\title{
DISSEMINATED INTRAVASCULAR COAGULATION (DIC) IN OBSTETRIC PRACTICE
}

\author{
SULTANA $\mathrm{S}^{1}$, BEGUM A ${ }^{2}$, KHAN MA ${ }^{3}$
}

\begin{abstract}
:
Disseminated intravascular coagulation (DIC) is an acquired and complex disorder that occurs in a wide variety of clinical conditions. This is basically a state of increased propensity for clot formation triggered by a variety of stimuli related to such diverse disorders as sepsis, endothelial cell damage (heat stroke, shock), obstetrical complication (abruptio placenta, amniotic fluid embolism, severe preeclampsia and retained intrauterine dead foetus) and neoplasm. DIC is a classic complication of obstetric conditions occurring in more than 50 percent of patients with obstetric causes. In DIC, an unregulated thrombin explosion cause release of free thrombin into the circulation that leads to the clinical features of DIC, with thrombin and plasmin responsible for the thrombotic and haemorrhagic manifestations, respectively. The diagnosis and treatment of this syndrome require an understanding of its pathophysiology, awareness of the disorders that can trigger it and its early recognition. Acute DIC is usually associated with infections, the commonest cause, about 10-20\% of patients with gram negative sepsis have evidence of DIC. Chronic DIC is usually associated with retained dead fetus, carcinomatosis. The diagnosis of this syndrome is essentially clinical, with laboratory tests providing confirmatory evidence. Microvascular thrombosis is the primary mechanism in most cases, and end organ failure is a major cause of death. No single diagnostic test exists for DIC. DIC is initially suggested by the following combination; a clinical condition consistent with DIC, thrombocytopenia, prolonged PT, APTT, and presence of FDP/D-dimer. Medical treatment depends on the cause of the DIC. Basically it involves removing the cause for example, delivery of placenta if it is retained or abrupted, delivery of foetus if retained, quick delivery if severe eclampsia and so on, hysterectomy if bleeding can not be controlled from placental site. After then, and/or con-currently treat DIC with blood and plasma transfusions and appropriate supportive measures. As the sequel of DIC can be devastating, early clinical suspicion and laboratory diagnosis are essential. This review article provides essential guideline for the appropriate diagnosis and clinical management of DIC in obstetric patients.
\end{abstract}

Key words: Disseminated intravascular coagulation (DIC), Obstetric, Thrombosis, Fibrin, Ddimer, FDP, Anticoagulant.

J Dhaka Med Coll. 2011; 20(1) : 68-74.

\section{Introduction:}

Disseminated intravascular coagulation is a clinical syndrome characterized by systemic activation of coagulation cascade simultaneously leads to intravascular thrombus formation (compromising blood supply to various organs ) and exhaustion of platelets and coagulation factors (resulting in hemorrhage). DIC is uncommon but not rare. The incidence of the syndrome varies in association with different disorders. In obstetrical conditions, such as abruptio placenta and amniotic fluid embolism, DIC occurring in more than $50 \%$ of cases $^{1}$. In severe preeclampsia DIC occurred in $7 \%$ of patients, in one study ${ }^{2}$. Disseminated intravascular coagulation may be classified according to its acuteness and clinical severity; i) Acute DIC, ii) Sub-acute DIC or Chronic DIC. Obstetrical causes of DIC can be classified as acute and chronic. DIC may be the result of single or multiple conditions. Acute DIC in placental abruption, amniotic fluid embolism, severe preeclampsia or eclampsia, Obstetric sepsis, Septic abortion or saline abortion, acute fatty liver of pregnancy, extensive surgical trauma, massive blood transfusion. sub-acute or chronic DIC in retained dead fetus syndrome, retained products of conception,

1. Dr. Sufia Sultana, Assistant Professor, Department of Obstetrics and Gynaecology, National Institute of Cancer Research \& Hospital (NICRH), Mohakhali, Dhaka.

2. Prof. Anowara Begum, Ex-Professor \& Head, Department of Obstetrics and Gynaecology, Dhaka Medical College \& Hospital, Dhaka.

3. Prof. MA Khan, Professor \& Head, Department of Haematology, Dhaka Medical College \& Hospital, Dhaka.

Correspondence: Dr. Sufia Sultana, Assistant Professor, Department of Obstetrics and Gynaecology, National Institute of Cancer Research \& Hospital (NICRH), Mohakhali, Dhaka. Cell Phone: +8801715867740, E-mail: drsufiasultana@gmail.com 
degenerating hydatidiform moles, abdominal (ectopic) pregnancy, saline induced abortion, fetomatarnal blood passage, postpartum haemolytic ureamic syndrom metastatic carcinoma (e.g; ovary). Hematological changes during pregnancy are critical. Normal pregnancy is associated with a range of alterations to haemostatic component, which combine to give an increased risk of hemorrhage, thrombosis and DIC. Pregnancy is associated with a $20-200 \%$ increase in level of fibrinogen and factors II, VII, X, VIII and XII, where as concentrations of factors $\mathrm{V}$ and IX are unchanged $^{\mathbf{3}}$. Thus net effect of pregnancy associated changes in haemostatic and fibrinolytic proteins enhances the risk of thromboembolism. The normal range for the platelet count (150- $400 \times 10^{9} / \mathrm{L}$ ) does not alter during pregnancy.

\section{The pathogenesis of DIC is complex ${ }^{4-8}$ :}

The mechanisms that trigger DIC act on processes of platelet adhesion and aggregation and contact activated (intrinsic) and tissue factor activated (extrinsic) pathway of coagulation. The normal compensatory process may become impaired, creating a self perpetuating "vicious cycle". The ultimate outcome is determined by a dynamic interplay between the various pathologic processes and compensatory mechanisms in other words fibrin deposition versus fibrinolysis; depletion versus repletion of coagulation of factors and platelets; and production versus clearance of fibrin, FDP and other products of coagulation. In most forms of DIC, the initiating factors are multiple and interrelated. Acute DIC only results when the normal compensatory mechanisms of haemostasis have been overcome. Direct platelet activation in septicemia and viraemic states; platelet activation will also result from vessel wall endothelial damage and following thrombin generation by the coagulation cascade. A combination of coagulation factor deficiency, thrombocytopenia, impaired platelet function, and inhibitory action of raised FDPS, causes the generalized and continuing widespread bleeding tendency. Chronic or "compensated" DIC result from a weak or intermittent activating stimulus. In such patients, destruction and production of coagulation factors and platelets are balanced. The pathophysiology of such chronic, sub-acute or "compensated" DIC is fundamentally the same as that in the acute case. Nevertheless, the distinction is valuable because the clinical picture and laboratory findings in the chronic form are quite variable and may be diagnostically confusing. Chronic DIC has been described in most patients with intrauterine foetal death. Other etiologic factors that may produce chronic DIC include various forms of vasculitis, hydatidiform moles, and degenerating leiomyomas. Chronic DIC has many clinical and laboratory features that resemble an intermediate stage between the "hypercoagulable state" and acute DIC. Indeed, even normal pregnancy has been suggested as a form of low grade "physiologic" DIC which at term becomes overt for a short time.

The exposure of procoagulent tissue extracts to blood is a major contributory factor in most forms of DIC and is of major pathogenetic importance in cases associated with abruption placenta, intrauterine fetal death, acute promyelocytic leukaemia, amniotic fluid embolism, massive trauma, and various neoplasms.

Abruptio placentae: In about 10 percent of patients with abruptio placentae, an overt coagulopathy develops due to DIC and fibninolysis ${ }^{9}$. Degree of placental separaton correlates with the severity of DIC. DIC may occur in placental abruption involving liberation of tissue thromboplastin or possible intrauterine consumption of fibrinogen and coagulation factors during the formation of retro- placental clot. This leads to activation of the extrinsic coagulation mechanism.

Amniotic fluid embolism: Here is some uncertainty about what elements in the amniotic fluid are responsible for the syndrome and whether prior exposure and sensitization to amniotic fluid are necessary. Cellular elements and other debris, such as lanugo hair and vernix, may be important. Mucin from meconium in the amniotic fluid causes a particularly intense reaction in the pulmonary arterioles ${ }^{10}$. The manifestation of amniotic 
fluid embolism does not appear to the dependent on the quantity of amniotic fluid that enters the maternal circulation or reaches the pulmonary circulation and therefore it is believed to be an anaphylactic response which is patient depenent ${ }^{11}$. The pathophysiology of amniotic fluid embolism is a biphasic response: the initial phase occurs after the amniotic fluid enters the pulmonary circulation resulting in vasoconstriction and pulmonary hypertension; second phase is the stage of hemodynamic compromise characterized by oedema and right sided heart failure. Amniotic fluid contains high concentrations of plasminogen proactivators, but it lacks plasminogen activator.

Intrauterine foetal death: Retention of a dead foetus can precipitate abnormal maternal bleeding. Unless the dead foetus is retain for three weeks or longer, clinically evident coagulation disorders are rare $^{12}$. The DIC associated with the foetal death is a chronic process, and the patient may present in either a compensated or a decompensated state. In intrauterine foetal death tissue thromboplastic substances from the dead foetus are slowly but continuously absorbed, producing a picture of chronic, but progressive DIC.

Preeclampsia \& Eclampsia: The exact etiology and mechanism remain unknown. This condition is associated with chronic coagulation abnormalities that may lead to thrombocytopenia and elevation of fibrin degradation products. The coagulation abnormalities reflect platelet adherence to exposed collagen at the sites of damage endothelia. It is related to, but clearly distinct from DIC. Preeclampsia and eclampsia are unknown and unclear precursors to DIC. In preeclampsia and eclampsia, patients have higher amounts of FDPs in the blood and urine than others. The thrombocytopenia seen in preeclampsia and eclampsia is due to the increased consumption of platelets ${ }^{13}$.

Saline or septic abortion: Saline induced abortion has been associated with subclinical DIC ${ }^{14}$. Severe cases of DIC have occurred in 1:400-1:1000 cases. Disease may be related to the release of tissue thromboplastin from the placenta. Septic abortion may also cause release of tissue thromboplastin or release of bacterial endotoxin (phospholipids).The common infections in obstetric practice are septic abortion and chorioamnionitis in premature rupture of the membranes. Gramnegative bacteria are responsible for septic shock in more than 95 percent of affected patients.

Massive trauma: In massive trauma, major surgical procedures such as lacerated injuries of cervix during delivery, damaged tissue expressing tissue factor activity presumably is a major initiating factor ${ }^{15}$.

Infections: DIC often accompanies septicemia (mainly due to Gram-negative) as a result of septic abortion, bacteria that possess potent endotoxins.

Shock: It has been suggested that DIC involved in all forms of shock and that it is the central feature in irreversible or refractory forms.

\section{Clinical presentation:}

The major clinical features of DIC are bleeding, often of serious magnitude and abrupt onset, a variable element of shock that is often out of proportion to apparent blood loss, and symptoms of hypoperfusion of various vascular beds. Acute renal failure is common, and thromboembolic manifestations often are noted. Evidence of major organ dysfunction is a common finding in patients with acute DIC, most often including signs, symptoms, and laboratory evidence of abnormal pulmonary, renal, hepatic, and central nervous system function. Major clinical problem and presenting feature of acute DIC is bleeding, which can vary from mild to severe form, manifesting as generalized bruising, ecchimosis, petechiae, especially over dependent areas and pressure sites and or bleeding from previously intact venipuncture sites or around indwelling intravenous needles. In patients who develop DIC after surgical procedures, alarming haemorrhage may develop around drains and accumulation of blood may be concealed in serous cavities. In Obstetric cases, post partum bleeding from the vagina may be particularly severe. Severe haemorrhage and hypovolaemic 
shock can occur extremely rapidly in pregnancy, when it is usually related to labour. In subacute or chronic DIC, there is slow activation of the haemostatic system, with spontaneous bruising rather than major clinical bleeding episodes. Such as intrauterine foetal death may produce as slow progressive form of DIC, in which bleeding is a relatively late manifestation, often fore shadowed by deteriorating renal function.

\section{Laboratory Diagnosis:}

In acute DIC, it is essential to demonstrate the abnormal laboratory parameters accurately and quickly, so that appropriate therapy can be instigated. The main haemostatic abnormalities are reflected by a prolonged thrombin time, hypofibrinogenaemia and thrombocytopenia. A fibrinogen level below 100 $\mathrm{x} 10^{9} / \mathrm{L}$, and a thrombin time of more than double the control value are regarded as virtually diagnostic, especially if associated with a generalized bleeding state.

\section{Laboratory investigations}

(A) Basic blood Examination: Examination of the blood smear reveals schistocytosis in approximately $50 \%$ of cases $\mathbf{1 7}$. More subtle evidence of intravascular haemolysis often is found, such as increased serum level of lactic acid dehydrogenase (LDH) and diminished heptoglobin levels. Thrombocytopenia is an early and consistent sign of acute DIC Platelet counts in the range of 50 to $100 \times 10^{9} / \mathrm{L}$ are usual findings but thrombocytopenia may be severe.

(B) Coagulation defect: The Activated partial thromboplastin time (APTT), Prothrombin time (PT), Thrombin time (TT) are prolonged in most patients with acute DIC. Early in the course of the disorder and in chronic DIC, the APTT may be normal or even shorter than normal. The plasma levels of fibrinogen and of factor $\mathrm{V}$ and XIII usually are significantly depressed; fibrinogen and factor $\mathrm{V}$ are the most consistently affected ${ }^{18}$. In many patients particularly those with abruptio placentae, normal prothrombin levels are maintained 9,16 but marked hypoprothrombinaemia often is present in those with sepsis ${ }^{17}$. In patients with chronic DIC, prothrombin deficiency is unusual, where as significant deficiencies of factor $\mathrm{X}$ are often noted.

(C) Tests for fibrinolysis: Fibrinolytic activity causes raised level of circulating fibrin complexes and FDPs. Levels above $100 \mu \mathrm{g} / \mathrm{ml}$ are usually found in acute DIC (normal range< $10 \mu \mathrm{g} / \mathrm{ml}{ }^{18}$. Immunological assays based on monoclonal antibodies to D-dimer are more specific than a general screen. Since false positive results may be seen with FDPs. FDPs test is not diagnostic of DIC, yet levels are elevated in $85-100 \%$ of patients. The D-dimer test may be more specific in diagnosing DIC, especially in distinguishing the coagulopathy of liver disease from DIC 18,19.

Bed side tests: Bed side tests to evaluate the blood coagulation disorders, which can give useful information that help in tacking the crisis. Bed side tests that may be done are: i. Bleeding time ii. Clotting time, iii. Clot observation (Weiber) test. iv. Serial clot lysis test v. blood film.

Screening tests: Platelet count (decreased), Bleeding time (increased), Prothrombin time(increased), APTT(increased or variable), Thrombin time (increased), RBCmorphology (schistocytes \& microspherocytes)

\section{Differential diagnosis:}

DIC with obstetric causes, the clinical conditions which are most commonly giving similar clinical pictures are the followings:

1. HELLP syndrome

2. Acute Liver Failure / Acute fatty liver in pregnancy.

3. Idiopathic/Autoimmune Thrombocytopenic Purpura (ITP/ATP).

4. Thrombotic Thrombocytopenic Purpura (TTP)

5. Postpartum Haemolytic Uraemic Syndrome (PHUS)

6. Primary Fibrinogenolysis.

7. Acquired Hemophilia (Factor F-VIII inhibitor).

Essentials of Diagnosis: No single diagnostic test exists for DIC. DIC is initially suggested by the following combination: 
- History of recent bleeding diathesis, always secondary to some cause.

- Clinical evidence of multiple bleeding points associated with purpura petechiae and ecchymosis on physical examination.

- Laboratory findings classically include thrombocytopenia $\left(<100 \times 10^{9} / \mathrm{L}\right)$, prolonged PT, APTT and TT, and presence of FDP / DDimer. Other tests listed here also may help to exclude DIC.

\section{Management:}

DIC always is the end result of a serious underlying disorder. Although the patient may benefit greatly from measures directed at the DIC or from the replacement of depleted coagulation factors or platelets, cure of the syndrome depend on prompt and energetic treatment of the primary disorder. Symptoms of DIC, although sometimes indolent, can cause life threatening haemorrhage and may require emergency management. This includes:

\section{Detection and elimination of primary} cause: It is the cornerstone of management of DIC. Determine the underlying cause and attempt to correct it immediately.

II. Supportive measures: Measures to control the major symptoms, either bleeding or thrombosis.

III. Replacement and prophylactic regimen: Prevention of recurrence in cases of chronic DIC. Treatment will vary with the clinical presentation. In patients with an obstetric complication such as abruptio placentae, intrauterine dead foetus or acute bacterial sepsis, the underlying disorder is easy to correct, and prompt delivery of the foetus and placenta or treatment with appropriate antibiotics will reverse the DIC syndrome. Moderate and low grade DIC may not be associated with clinical evidence of excessive bleeding and often will require close observation but no further therapy. The condition will not resolve until the triggering factor is removed, and death of patient with DIC are often the result of the underlying disease.

Supportive therapy should be directed to the correction of shock, acidosis, and tissue ischemia. Cardiopulmonary support, including ionotropic therapy, blood replacement, and associated ventilation, should be implemented with the patient in close proximity to a delivery suite. Tissue perfusion and respiratory function must be maintained by replacing intravenous fluid and providing oxygen to correct hypoxia. Parental antibiotic should be administered without delay when bacterial infection is suspected. Foetal monitoring, careful recording of maternal fluid balance, and serial evaluation of coagulation parameters are extremely important. Patient may be treated with blood components to replace depleted coagulation factors, platelet, and natural inhibitors of thrombin and plasmin in an attempt to reduce bleeding while the underlying problem is corrected. Specific replacement of fibrinogen should be accomplished by cryoprecipitate. Each unit of cryoprecipitate carries approximately $200 \mathrm{mg}$ of fibrinogen. Platelet should only be administered in the face of active bleeding with a platelet count 20$30 \times 10^{9} / \mathrm{L}$ or less or following massive blood transfusion with stored old blood. Critically ill patients may develop a coagulopathy, because of vitamin $\mathrm{K}$ deficiency, and $10 \mathrm{mg}$ of vitamin $\mathrm{K}$ should be given on two consecutive days before coagulopathy is attributed exclusively to DIC 20. Patients with DIC can also become vitamin $\mathrm{K}$ deficiency because of it's increased use and administration of vitamin $\mathrm{K}$ to all patients with suspected DIC will replenish stores. Some doctors also give folic acid in order to prevent acute folate deficiency and impaired platelet production.

Vaginal delivery, without episiotomy if possible, is preferable to cesarean section. Failure of improvement in the coagulopathy within several hours after delivery suggests sepsis, liver disease, retained product of conception, or a congenital coagulation defect.

The use of heparin in the treatment of bleeding is still controversial. Although it is a logical way to reduce thrombin generation and prevent further consumption of clotting proteins, it should be reserved for patients with thrombosis or those who continue to bleed despite vigorous treatment with plasma and platelets. Patients who initially have mild DIC and may not be 
symptomatic may begin to bleed following surgery. For example, mild DIC, without clinical bleeding, has been documented during saline or prostaglandin-induced midtrimester abortions. Prophylactic treatment of patients with heparin may prevent progression of a mild DIC syndrome and in some patients a retained dead foetus that require surgical extraction. However most patients with low grade DIC can be managed simply with plasma and platelet replacement and do not require heparin.

\section{Emergency care:}

- Monitor vital signs: pulse, blood pressure, respiration, renal out put and level of consciousness.

- Document extent of haemorrhage and thrombosis.

- Determine the underlying cause of the DIC and initiate therapy.

- Send blood specimens for appropriate coagulation studies and other diagnostic laboratory tests. Screening test for DIC are: BT, PT, APTT, Platelet count, RBC morphology.

- Obtain appropriate imaging studies if necessary.

- Supportive measures are essential. Attend to life threatening issues such as air ways compromise or severe haemorrhage. Correction of fluid and electrolyte imbalance.

- Replace blood products as indicated: RBC transfusion (i.e. packed red cells), Platelet concentrate, Fresh frogen plasma (FFP). Cryoprecipitate.

\section{Consultation:}

- Consult hematologist for assistance with diagnosis and management.

- Consult critical care medicine if multiple organs failure is present.

- Consult transfusion medicine; determine the availability of general and specialized blood products that may be necessary for the acute management of fulminant DIC.

\section{Medication:}

Therapy should be based on etiology and aimed at eliminating the underlying disease. Therapy should be appropriately aggressive for the patient's age, disease, and the severity and location of haemorrhage/thrombosis. Treatment for acute DIC includes anticoagulants, blood components, and antifibrinolytics.

Haemostatic and coagulation parameters should be monitored continuously during treatment. Base therapeutic decisions depend on clinical and laboratory evaluation of haemostasis.

(A) Blood components: Blood components are used to correct abnormal haemostatic parameters. These products should be considered only after initial supportive and anticoagulant therapy.

i) Packed red blood cells (washed): Preferred to whole blood since they limit volume, immune, and storage complications. One unit PRBCs should raise haemoglobin by $1 \mathrm{~g} / \mathrm{dl}$ or raise haematocrit by $3 \%$.

ii) Platelet concentrates: Patient with DIC, bleeding and platelet counts less than $50 \times 10^{9}$ / L, should be considered for platelet transfusion. DIC patients may require a higher platelet count for adequate haemostasis then patients with thrombocytopenia in the absence of platelet dysfunction (in other conditions of thrombocytopenia seldom needed platelet transfusion until platelet count is less than $20 \times 10^{9} / \mathrm{L}$ ).

iii) Fresh Frozen Plasma (FFP): FFP contains all the coagulation factors and inhibitors, ATIII and protein-C. 4 to 5 units of FFP should be rapidly infused.

iv) Cryoprecipitate: It contain factor VIIIc, vWF, fibrinogen, fibronectin and some factor XIII. The amount of cryoprecipitate given should be sufficient to elevate the plasma fibrinogen level to at least $100-150 \mathrm{mg} / \mathrm{dl}$. As a general guide, $3 \mathrm{~g}$ of fibrinogen can be expected to raise the plasma level of an adult patient approximately $100 \mathrm{mg} / \mathrm{dl}$.

\section{B) Anticoagulants:}

These agents are used in the treatment of clinically evident intravascular thrombosis when the patient continues to bleed or clot 4-6 
hours after initiation of primary and supportive therapy. Take special precaution in obstetric emergencies or massive liver.

i) Heparin: The indications for treatment with heparin and the dose required are not established $^{21}$. There is no conclusive evidence that heparin treatment reduces mortality or morbidity in acute DIC. Heparin treatment may be ineffective because it requires antithrombin-III for anticoagulant activity, and this is usually reduced in DIC. There is one instance, however, where heparin has been demonstrated to benefit in pregnancy-related DIC. In the case of retained dead foetus with an intact vascular system, heparin may be administered to interrupt the coagulation process and thrombocytopenia for several days until safe delivery may be implemented 22,23

ii) Low molecular weight-heparin: It is increasingly used in pregnancy because of its favorable dosing regimen and lack of need for routine monitoring and lowers risks of haemorrhage, thrombocytopenia and osteoporosis. Antithrombin III and Protein-C are other promising potion.

(C) Antifibrinolytic agents: These are used only after all other therapeutic modalities have been tried and deemed unsuccessful. In theory, epsilon-aminocaproic acid (EACA) and other antifibrinolytics agents (Tranexamic acid) remove the major antagonist of intravascular fibrin formation, and in several well documented cases of DIC, their use has resulted in serious and even fatal thromboembolic complication. The indiscriminate use of such drugs should be discouraged.

\section{References:}

1. Weiner $\mathrm{C}$ P. The obstetric patient and disseminated intravascular coagulation. Clin Perinatal 1986; 13: 705.

2. Sibai BM, Spinnato JA, Watson DI, Hill GA, Anderson GD. Pregnancy outcome in 303 cases with severe preeclampsia. Obstet Gynecol 1984; 64: 319-25.

3. Delorme MA, Burrows RF, Ofosu FA, et al. Thrombin regulation in mother and fetus during pregnancy. Semin Thromb Hemost 1992; 18: 81-90.

4. Bick RL, Adams T. Disseminated intravascular coagulation: etiology, pathophysiology, diagnosis and management. Med Counterpoint 1974; 6: 38.

5. Plow EF. Leukcyte elastase release during blood coagulation: a potential mechanism for activation of the alternative fibrinolytic pathway. J Clin Invest 1982; 69: 564-72.

6. Mekay DG. Trauma and disseminated intravascular coagulation. J Trauma. 1969; 9: 646-60.

7. van Glinket CJ, van Aken WG, Oh JIH, Vrecken J. Stimulation of monocyte progualent activity by adherence to different surfaces. Br J Haematol 1977; 37: 35-45.

8. McGrath JM, Stewart GJ. The effects of endotoxin on vascular endothelium. 1969; 129: 833-48.

9. Sher G. Pathogenesis and management of uterineinertia complicating abruption placentae with consumptive coagulopathy. Am. J. Obstet Gynecol 1977; 129: 164-70.

10. Chatelain SM, Qilirk JG. Amniotic fluid and thromboembolism. Clin Obstet Gynecol 1990; 33: 478.

11. Clark SL, Montz FJ, Phelan JP. Hemodynamic alterations associated with amniotic fluid embolism: a reappraisal. Am J Obstet Gynecol 1985; 151: 617-21.

12. Tricomi V, Hohl SG. Fetal death in utero. Am J Obstet Gynecol 1957; 74: 1092.

13. Kleckner HB, Gites HR, Corrgan JJ. The association of maternal and neonatal thrombocytopenia in high risk pregnancies. Am J Obstet Gynecol 1977; 128: 235-8.

14. Cohen E, Ballard CA. Consumption coagulopathy associated with intra-amniotic saline instillation and the effect of oxytocin. Obstet Gynecol 1974; 43: 300 .

15. Attar S, Boyd D, Layne E, et al. Alterations in coagulation and fibrinolisis mechanisms in acute trauma. J Trauma 1969; 9: 939-65.

16. Merskey C, Johnson AJ, Kleiner GJ, Wohl H. The defibrination syndrome: Clinical fearures and laboratory diagnosis. Br J Haematol 1967; 13: 52849.

17. Corrigan JJ, Jordan CM. Heparin therapy in septicemia with disseminated intravascular coagulation. N Engl J Med 1970; 283: 778-82.

18. Carr J M, McKinney M, Mc Donagh J. Diagnosis of disseminated intravascular coagulation: Role of D- dimmer. Am J Clin Pathol 1989; 91: 280-7.

19. Alperin J. Coagulopathy caused by vitamen K deficiency in critically ill, hospitalized patients. JAMA 1987; 258: 1916-9.

20. Fenstein D. Diagnosis and management of disseminated intravascular coagulation: the role of heparin therapy. Blood 1982; 60: 284-7.

21. Wessler S. Gitel SN. Heparin: new concepts relevant to clinical use. Blood 1979; 53: 525-44.

22. Glinsberg J, Kowalchuk G, Hirch J, et al. Heparin therapy during pregnancy: risk to the fetus and mother. Arch Intern Med 1989; 149: 2233-6.

23. Feigin MD, Lounwood DL. Low molecular weight heparin and their use in obstetrics and gynecology. Obstet Gynecol Surv 1994; 49: 424-31. 